\title{
Efrat Eizenberg
}

\section{Soziale Proteste in Israel: die Eroberung neuer sozialer Räume}

Der größte Teil der israelischen Bevölkerung lebt an der Küste. Hier erreicht der Sommer mit starker Hitze, brennender Sonne und extremer Feuchtigkeit seinen Höhepunkt Mitte Juli. In dieser Zeit sind die Straßen leergefegt und das Leben erwacht erst nach Sonnenuntergang (und selbst dann bleibt die Feuchtigkeit bestehen). Aber Mitte Juli 2011 war alles anders: Viele Menschen zogen sich nicht wie sonst in klimatisierte Räume zurück, sondern gingen auf die Straße, um zu protestieren, um den Widerstand aufzubauen, um eine Revolution einzuleiten.

Der israelische Sommer folgte dem europäischen (in Griechenland und Spanien) und dem arabischen Frühling (in Tunesien, Ägypten, Syrien und Libyen), aber er stellte ursprünglich keine Fortsetzung dieser Bewegungen dar, wie wir das später in den USA erleben konnten. Er wurde durch zwei, eigentlich miteinander konkurrierende Konsumentenproteste ausgelöst, die bis dahin nicht zum Repertoire der politischen Kultur gehört hatten. Zunächst gab es einen Konsumentenboykott gegen Hüttenkäse, dessen Preis (ebenso wie derjenige anderer Milchprodukte) erhöht worden war. Er war insofern erfolgreich, als die Preiserhöhungen zurückgenommen wurden. Kurz darauf stellten mehrere junge Leute mitten in Tel Aviv Zelte auf, weil sie sich nicht in der Lage sahen, zu einem erschwinglichen Preis eine passable Wohnung zu bekommen. Nach und nach gesellten sich andere zu ihnen, alles junge Menschen aus der Mittelschicht, die als Mieter von demselben Problem betroffen waren. Für einige Tage sah dieses Stadtviertel mit seinen leicht bekleideten Jugendlichen, mit Musik und Zigarettenrauch in der Luft wie die urbane Version eines Club Méditerrannée für Singles, Junge und Schöne aus.

Was bis dahin ein jahrelang unterdrücktes Rumoren im Untergrund gewesen war, brach nun als sozialer Kampf aus und brachte hier wie in anderen Städten Menschen zusammen, die sich nach ihrem Alter, ihrer sozialen, ethnischen und religiösen Zugehörigkeit unterschieden - eine multitude im Sinn von Hardt und Negri (2004). Wogegen protestierten sie in Israel, in einem demokratischen Land, dessen Wirtschaft bis dahin stark genug war, um sich der globalen Krise zu entziehen? In der ersten Woche protestierten sie gegen überteuerte Mieten und 
den Mangel an bezahlbarem Wohnraum sowie gegen das Fehlen von Regelungen zum Mieterschutz, was zu einem drastischen Steigen der Mieten in allen Teilen des Landes geführt hatte. In der zweiten Woche des Protestes erweiterte sich das Themenspektrum auf sämtliche Kosten für Wohnen und Lebenshaltung. Doch schrittweise kam eine tieferliegende kollektive Empörung zum Ausdruck und die Proteste wurden zu einem Ruf nach sozialer Gerechtigkeit - nach sozialer Gerechtigkeit, die eine Systemveränderung, eine Verschiebung der sozialen Prioritäten, ein Infragestellen der neoliberalen Politik und die Herstellung (oder Wiederherstellung) sozialer Demokratie bedeuten würde.

Vom arabischen Frühling übernahmen die israelischen Protestierer das Skandieren von Parolen, aber es gab keine ausdrückliche Forderung nach dem Rücktritt der politischen Führung, sondern nach einer anderen Politik: „Das Volk verlangt soziale Gerechtigkeit“. Aus Spanien übernahmen sie die Methoden der öffentlichen Diskussionen und der Räte. Im Anschluss an die arabischen und an die europäischen Revolten wurden auch hier Camps errichtet, die das öffentliche Bild der Bewegung prägten und es gab wöchentliche Protestmärsche. Wie anderswo wurde dieses soziale Erwachen abwechselnd als „Protest“, „Widerstand“ oder als „Revolution“ bezeichnet, und dies sowohl auf der Straße selbst wie in den Medien. Aber was ist es nun tatsächlich? Als was ist diese gewaltlose, aber andauernde Intervention von Bürgern in die öffentliche Sphäre zu verstehen?

Für mehr als zwei Monate gab es mehr als 60 äußerst aktive Camps in den Zentren oder auch am Rand von israelischen Städten. In ihnen diskutierten die Zeltbewohner und ihre Besucher soziale Fragen, hörten Vorlesungen von Akademikern, entwickelten gesellschaftliche Alternativen, schufen Kunstwerke, tanzten, musizierten oder improvisierten Theater-Aufführungen und stellten (nahezu) jede Woche eine Demonstration auf die Beine. Delegierte der verschiedenen Camps wurden zu einem Lenkungsgremium entsandt, das über gemeinsame Aktionen entschied und diese ebenso wie die Medienauftritte plante. Politiker wurden allgemein als personae non gratae angesehen (außer sie kamen als Privatpersonen wie andere auch) und Geldspenden wurden, anders als Sachspenden und Arbeitsleistungen, nicht angenommen.

Das Hauptquartier des Protests befand sich in Tel Aviv auf dem Rothschild Boulevard, auf einer der größten Straßen der Stadt, an der auch die Zentralen des Finanzsektors ansässig sind. Auf diesem Luxus-Boulevard ereignete sich ein permanentes Festival - ein festliches politisches Ereignis, die nicht-kommerzielle Feier einer wiederentdeckten sozialen und einer neuorganisierten öffentlichen Sphäre. Dies geschah in erster Linie und vor allem dadurch, dass man sich die Straßen zurückholte und sich den Raum für alternative, nicht-kommerzielle Zwecke aneignete: es gab öffentliche Diskussionen, öffentliche Vorlesungen und Kunstereignisse aller Art. Zu dieser Zeit wurde der Boulevard somit zur interes- 
santesten und kreativsten Kunstszene des Landes. Für eine bedeutende Periode entstand ein Raum der Alternativen, der sich deutlich von seiner Umgebung in dieser global city abhob. Hier entfalteten sich gesellschaftliche Interaktionen der Zivilität, der Solidarität, der Kooperation und der Unterstützung, die Erfahrungen eines besseren und wünschenswerten Reichtums vermittelten.

Diesespolitifest ${ }^{1}$ dauerte über zwei Monate. Die hohen Feiertage Ende September und behördliche Anordnungen der Stadt vertrieben die meisten Camp-Bewohner, so dass die übrig gebliebenen Zelte nunmehr von Obdachlosen bewohnt wurden. Starke Regengüsse im November zwangen viele von ihnen dazu, Kompromisse einzugehen und in Übergangswohnungen einzuziehen oder einmalige Unterstützungszahlungen der Stadt anzunehmen. Am Ende des Monats gab es kaum noch physische Spuren der größten Proteste, die Israel jemals gesehen hat. Die Stadtverwaltung beeilte sich, die Flächen des Boulevards, auf denen die Zelte gestanden waren, mit Gras zu bepflanzen, um, wie der Bürgermeister verkündete, „den Rothschild Boulevard dem Bereich der Öffentlichkeit zurück zu geben“, nachdem die Polizei ausgerückt war, um die Zelte abzuräumen. Um diese gegensätzlichen Definitionen des „Bereichs der Öffentlichkeit” ging es bei den sozialen Auseinandersetzungen.

Wie können wir das definieren, was in Israel stattgefunden hat? Was wären die Kriterien, um es als Protest, als Widerstand oder Revolution zu bezeichnen? Sind es Gewalt und Verletzte, die aus einem Protest Widerstand machen? Sind es die Dauer oder die aufgeworfenen Themen - spezifische oder allgemeine soziale Probleme, diejenigen bestimmter Gruppen oder der Mehrheit der Bevölkerung? Oder geht es um den Erfolg der Kämpfe? Und was würde dann als Erfolg gelten können? Die Tatsache, dass die politische Führung ausgewechselt würde oder neue Gesetze und Prinzipien verabschiedet würden? Müsste eine grundsätzliche Veränderung stattfinden, damit man von einer Revolution sprechen kann oder könnte man bereits darin einen Sieg sehen, dass das Tempo der Veränderung verlangsamt würde, wie Polanyi es vertrat (2001: 39)? Bei den israelischen Protesten wurden keine grundsätzlichen Veränderungen des Sozialen verlangt. Die Forderungen gingen dahin, das System zu verändern, nicht es durch ein anderes zu ersetzen. Der Aufschrei kam aus dem Inneren des Kapitalismus.

Auf den ersten Blick könnte man meinen, die sozialen Kämpfe seien zu einem Ende gekommen, dennoch können einige greifbare Erfolge festgestellt werden, sie werden mit anderen Mitteln fortgesetzt. Eine ihrer Wirkungen besteht darin, dass der Premierminister ein Regierungskomitee - das Trachtenberg-Komitee

1 Eine Form der Verbindung von öffentlichen politischen Diskussionen und Feiern als Wiederbelebung früherer Gepflogenheiten, als Politiker in den USA auf Jahrmärkten auftraten - fand unter diesem Namen erstmals 2011 in San Diego, Kalifornien, statt. 
- einberufen hat, das die Forderungen der Protestierenden untersuchen sollte und tatsächlich eine Reihe von Empfehlungen erarbeitet hat, denen seitens der Regierung mehrheitlich zugestimmt wurde. Wenngleich diese Empfehlungen die Vorstellung der sozialen Demokratie nur ansatzweise weiter entwickeln, wurden sie öffentlich vorgestellt und in den Medien breit rezipiert. Professor Trachtenberg, ein weithin geschätzter Ökonom, nahm eine Analyse des aktuellen Gesellschaftssystems vor, wie man sie bis dahin nicht gekannt hatte und in welcher der üblicherweise marginalisierte kritische Diskurs über Ausbeutung und wachsende soziale Ungleichheiten, der bis dahin stets als „kommunistisch“ oder „sozialistisch“ diskreditiert wurde, als faktisch zutreffend und allgemein anerkannt präsentiert wurde. Das bedeutete, dass die Forderungen der Protestierenden nunmehr legitimiert und gerechtfertigt erschienen, während man diese selbst zuvor gerne als verwöhnt und verweichlicht dargestellt hatte. So bestätigte die Analyse ihre eigenen Schilderungen der aktuellen Verhältnisse und letztlich konnte keine politische Partei (selbst nicht die marktgläubigste) dieser Lesart der sozialen Schäden einer entfesselten Marktökonomie widersprechen. Das Fehlen eines Widerspruchs oder selbst die Zustimmung zu einer derartigen Darstellung der Lage ist keineswegs als Indikator für einen durchschlagenden Erfolg zu sehen, sondern bedeutet lediglich, dass neoliberale Politiker in der Lage sind, Umfragen zu lesen. Ihnen zufolge stimmten $87 \%$ der Befragten den Protesten zu.

Ein weiterer Erfolg besteht darin, dass die Gewerkschaft Histradut (die für die Arbeitnehmer im öffentlichen Dienst zuständig ist) neue Anstöße erhielt, Veränderungen durchzusetzen. Histradut stellte einstmals einen entscheidenden Akteur beim Ausbalancieren der Marktkräfte dar (Shalev/Grinberg 1989), hatte sich während vieler Jahre jedoch auf eine Rolle als Zaungast des Geschehens zurück gezogen und blieb bei dieser abwartenden Haltung auch, als auf den Straßen protestiert wurde. Mit ihrer bisherigen Passivität und ihrer Unterstützung der Regierung ist die Gewerkschaft mitverantwortlich für den jämmerlichen dritten Platz, den Israel im Ranking der OECD-Länder nach dem Ausmaß der sozialen Ungleichheit einnimmt (Divided we stand: Why Inequality Keeps Rising, 2011). Nachdem der Widerstand Histradut vom Zaun mitten ins soziale Geschehen gestoßen hatte, nahm sich die Gewerkschaft als erstes des Problems der Zeitarbeit an, also der Arbeitskräfte, die befristet beschäftigt und unorganisiert sind. Sie führt nun die Kämpfe gegen derartige Arbeitsverhältnisse im öffentlichen Sektor an und setzt sich auch im privaten Sektor für Verbesserungen ein. Wegen der unzureichenden Regulierung und der Missachtung selbst bestehender Vorschriften hat Israel beim Umfang der prekären Beschäftigung innerhalb der OECD-Länder inzwischen sogar den Spitzenplatz eingenommen. Die Gewerkschaft drohte mit einem Generalstreik und zwang das Finanzministerium zu Verhandlungen. 


\section{Das Soziale fordern}

Es gibt auch noch weitere, weniger greifbare Erfolge, die eher symbolischen Charakter haben, als dass sie tatsächlich zu gesellschaftlichen Veränderungen geführt hätten. Israel stellt eine Ethnokratie dar und insofern ist der politische Diskurs von den Spannungen zwischen der herrschenden Gruppe und der Minderheit geprägt. ${ }^{2}$ Die Besetzung der West-Bank und des Gaza-Streifens im Zuge des Krieges von 1967 vertiefte diese Spannungen und weitete sich zum blutigen IsraelPalästina-Konflikt aus. Eine Folge davon war, dass die Zugehörigkeit zu einer politischen Partei sich seither in erster Linie um die Frage dreht, welche Haltung diese zu den besetzten Gebieten und zu dem Konflikt einnimmt. Die Bevölkerung ist daher gespalten in linke Zionisten (die sich gegen die Besetzung und die Besiedlung der besetzten Gebiete wenden), rechte Zionisten (die an der Vorstellung eines Groß-Israels festhalten), und in einige kleinere arabische Parteien, die zur extremen Linken gehören und noch nie an der Regierung beteiligt waren. Diese Spaltung hat nicht nur dazu geführt, dass die politische Sphäre sich in zwei Lager aufgeteilt hat, die durch keinerlei Brücke verbunden sind, sondern auch dazu, dass der Israel-Palästina-Konflikt alle anderen Diskussionen überlagert hat. Dadurch konnte sich das neoliberale Projekt ungestört von öffentlicher Aufmerksamkeit etablieren, und zwar mit einer solchen Intensität, dass Israel - wie weiter oben erwähnt - zu einem der führenden Länder hinsichtlich sozialer Ungleichheit wurde. Die Protestbewegung stellte den hegemonialen politischen Diskurs in Frage und erklärte sich selbst als apolitisch, was heißen sollte, dass sie nicht an eine Partei gebunden und nicht in das gängige rechts-links-Schema einzuordnen war, sondern soziale Gerechtigkeit anstrebte. Ihre führenden Köpfe erklärten, dass sie nicht die Regierung auswechseln oder den Premierminister durch einen anderen ersetzen, sondern dass sie das gesellschaftliche System verändern wollten, wer immer dies auch durchsetzen würde. Diese Forderungen wurden durch die Regierung verworfen und die Protestierer als Anarchisten, Kommunisten oder einfach als Linke diffamiert. Doch die Tatbestände der Ungleichheit und der gebrochenen Solidarität wurden quer durch die Parteien zum Thema und selbst wenn die Mehrheit der engagierten Protestierer gebildete und säkular orientierte Angehörige der Mittelschicht waren, so kamen bald auch andere dazu. Camps wurden auch in armen Unterschicht-Vierteln errichtet, arabische Israelis beteiligten sich bis zu einem gewissen Grad am Widerstand, auch orthodoxe Juden stellten Zelte auf und beteten dort, selbst Vertreter der ultrarechten Siedler, die

2 Mit Ethnokratie ist ein politisches Regime gemeint, das Chancen und Rechte nach der Zugehörigkeit zur dominanten ethnischen Gruppe gewährt. Es ist weder demokratisch noch autoritär definiert (Yiftachel 2006). 
sich ursprünglich als Ursache vieler sozialer Nöte angeklagt sahen ${ }^{3}$, schlossen sich den Protestierenden mit der Begründung an, sie seien Teil der israelischen Gesellschaft und somit ihrer Kämpfe. Auch mit Hilfe einer starken Unterstützung durch die Medien war es möglich, das Soziale (oder zumindest 99\% davon) rund um ein kollektives Anliegen neu zu definieren und zu befestigen: somit wurde eine Diskussion wieder aufgenommen, die seit Jahrzehnten eingeschlafen war: die Diskussion darüber, in welcher Gesellschaft die Israelis eigentlich leben wollen. Diese Diskussion über das Soziale und über das grundsätzliche Verständnis von Gerechtigkeit schloss - und ich sollte sagen: bewusst - die Ungerechtigkeiten aus, die man den Palästinensern in den besetzten Gebieten vierzig Jahre lang angetan hatte. Dennoch handelte es sich um wichtige Diskussionen über Fragen der Demokratie, der Partizipation der Bürger, der Solidarität, der Gleichheit und anderer humanistischer Prinzipien - Diskussionen, die man bis dahin als ein Privileg anderer Gesellschaften angesehen hatte, die sich scheinbar erübrigten, wenn es um Frage des staatlichen Überlebens ging.

\section{Ein neuer Sozialcharakter}

Der soziale Wandel wurde diskursiv und praktisch eingeleitet, er bezog sich auf Ideen wie auf Aktionen. Ähnlich wie dies andere Widerstandsbewegungen taten, bediente sich auch der israelische Protest kolonialer Taktiken der Besetzung von Räumen. Die Protestierenden verwendeten dazu Zelte und Matten, Wohnzimmer-Zubehör wie Tische und Sofas, aufblasbare Schwimmbecken (um sich in der Sommerhitze abzukühlen), auch Camping-Busse ${ }^{4}$ - und ihre eigenen Körper.

Innerhalb dieser angeeigneten Räume entstand ein neuer und alternativer sozialer Raum. Dieser diente vor allem dazu sich auszudrücken: Ideen, Bedürfnisse und Wünsche zu formulieren, und zwar in kleinen oder großen Foren, bei Vorlesungen, bei allgemein zugänglichen öffentlichen Diskussionen und über das „Radio des Volkes”, auch über alle Arten von Kunst und Bewegungen des

3 Die Siedlungen in den besetzten Gebieten wurden kritisiert, weil sie als Verkörperung der sozialen Demokratie auftraten, die ursprünglich innerhalb der „Grünen Linie“ (den Außengrenzen vor dem Krieg von 1967) realisiert werden sollte, was aber faktisch durch die erheblichen Subventionen für diese Siedlungen verhindert wurde.

4 Campingbusse auf dem Rothschild Boulevard abzustellen bedeutete, die Taktik der Siedler zu imitieren, die genauso vorgehen, um neue Gebiete zu besetzen. Zunächst werden mehrere Campingbusse aufgestellt, meist in der Nacht. Am nächsten Morgen sieht das bereits wie eine Siedlung aus und man kann daran gehen, feste Bauten zu errichten. 
Körpers, so wenn man ging, lief, Rad oder Auto fuhr und sich mit Transparenten durch die Stadt bewegte, Parolen rief oder hupte.

Außerdem wurden große Anstrengungen unternommen, um alternative Räume zu schaffen, in denen jede und jeder die Möglichkeit haben sollte sich ausdrücken. Denn hier wurde der wesentliche Mangel der öffentlichen Sphäre in Israel gesehen, in der eine politische Kultur des divide et imperia herrscht, und in der die Trennungslinien nicht nur zwischen Linken und Rechten, sondern auch zwischen Religiösen und Befürwortern der Säkularisierung, zwischen Juden und Arabern wie auch zwischen Angehörigen verschiedener ethnischer Gruppen bestehen. Diese vielfältigen Fraktionen der israelischen Gesellschaft lernten, die Sicht zu überwinden, „die anderen seien Schuld an den eigenen Problemen und unzureichenden Geldmitteln“. Die Protestierer setzten sich für eine mutige und revolutionäre Rekonstruktion des Sozialen ein, wenn sie sagten, es ginge nicht um „eure“ gegen „unsere“ Gruppe, sondern darum, ein Gesellschaftssystem zu überwinden, das uns alle verletzt. Niemand sollte aufgrund seiner Zugehörigkeit zu einer Gruppe ausgeschlossen werden. Die Transformation sollte sich somit nicht nur darauf beziehen, die Wirtschaft wieder in den Dienst der Gesellschaft zu stellen (statt dies, wie bei dem „heiligen“ Ziel des Wachstums, genau andersherum zu sehen), sondern auch darauf, eine neue Vision von Gesellschaft zu entwickeln.

Die Gruppe von jungen Erwachsenen, die das Camp am Rothschild Boulevard initiiert hatte, wurde in der Öffentlichkeit als Anführer der sozialen Proteste angesehen. Man unterstützte sie oder wandte sich gegen sie und sie wurden auch persönlich diffamiert. Häufig wurden sie interviewt, hielten politische Ansprachen bei den wöchentlichen Kundgebungen und wurden zum „Gesicht“ des Widerstands. Doch wiesen sie die Rolle der Anführer zurück und betonten, dass sie lediglich die Probleme benennen, denen sich die meisten Israelis in ihrem täglichen Leben gegenüber sehen. Da es keine selbsternannten Anführer gab, waren die Proteste tatsächlich als jedermanns Proteste gesehen worden. In diesem Sinn lehnte es diese Gruppe auch ab, Einladungen der Regierung oder des Premierministers zu gemeinsamen Beratungen anzunehmen, da sie vertraten, es liege nicht an ihnen zu entscheiden, welche Veränderungen ausreichend wären. Stattdessen verkündeten sie, die Proteste auf den Straßen würden so lange weiter gehen, bis ausreichende Veränderungen erreicht würden.

Das einzige „offizielle“ Gremium, das den Widerstand repräsentierte, war das „Fachkomitee“ von 60 Akademikern - überwiegend Sozialwissenschaftler - das als Alternative zu dem von der Regierung eingesetzten Trachtenberg-Komitee fungieren sollte (in dem sich überwiegend Ökonomen befanden). Auf der Grundlage von öffentlichen Debatten und online-Stellungnahmen stellte dieses „Fachkomitee" einen alternativen Report zusammen. Die Diskussionen dazu und über daraus folgende praktische Empfehlungen in Versammlungen und an runden 
Tischen dauern an, doch fanden sie keinen Eingang in die Überlegungen der Exekutive, sie blieben auf den Bereich der Öffentlichkeit beschränkt.

Nach wie vor gibt es eine heftige Debatte darüber, wem der Widerstand "gehört". Anders als in den USA, wo die Proteste von Anfang an als der Aufstand von 99\% der Gesellschaft gegen das eine Prozent, das über Macht und Ressourcen verfügt, definiert wurden, war in Israel offensichtlich, dass die weiße aschkenasische Mittelklasse hier an der Spitze stand. Bis jetzt gibt es Klagen darüber, dass die unteren Klassen ausgegrenzt und vergessen, ihre „schäbigen“ Bedürfnisse nicht angesprochen wurden. Gleichwohl gab es auch Camps an den Stadträndern, in denen obdachlose Familien Unterschlupf fanden und deren Auflösung durch größere Menschenmengen verhindert wurde. Wenn sich bisher auch keine dauerhafte Lösung für sie abzeichnet, so trugen diese Aktionen zumindest dazu bei, dass ihre Probleme öffentliche Aufmerksamkeit erfuhren.

Die soziale Unruhe, durch welche die Histradut wiederbelebt wurde und die dazu führte, dass die Gewerkschaft den Kampf gegen eine der bedrückendsten Erscheinungen des ausbeuterischen Neoliberalismus, die Zeitarbeit, aufnahm, lenkte die Aufmerksamkeit auch auf andere Konflikte, deren Themen bisher eher unbeachtet geblieben waren. Dazu gehört die von orthodoxen Juden zunehmend betriebene Ausgrenzung von Frauen aus öffentlichen Räumen - wenn diese etwa nur bestimmte Busse benützen oder bestimmte Sitze in Bussen einnehmen oder auch in der Öffentlichkeit nicht als Sängerinnen oder Rednerinnen auftreten sollen, sobald sich orthodoxe Juden im Publikum befinden. Derartige Einschränkungen sind nicht neu, werden nun aber nicht mehr toleriert oder ignoriert. Ein anderes Beispiel ist das der äthiopischen Migranten, die besonders schlecht integriert wurden, was keineswegs neu ist; aber diese marginalisierte Gruppe trägt nun ihre kollektiven Probleme protestierend auf die Straße. Angestoßen durch die Erfahrungen der Solidarität im Sommer 2011 treten seither verschiedenste Fraktionen der Gesellschaft mit Aktionen in die Öffentlichkeit. Es gibt Proteste und stürmische Diskussionen, die auch durch die Medien in Gang gehalten werden, in denen Fragen der Gleichheit, Gerechtigkeit und Demokratie nach wie vor im Mittelpunkt stehen.

Die soziale Unruhe hat viele Gesichter. Radikal rechte Siedler und ihre Unterstützer treten als Gegenbewegung zu links-sozialdemokratischen Forderungen auf und terrorisieren in verstärktem Maß arabische Israelis und Palästinenser, also die arabische Bevölkerung innerhalb der „Grünen Linie“ wie auch in den besetzten Gebieten. Sie attackierten ihre Moscheen, Autos und Plantagen. Einige von ihnen drangen sogar in ein Armeelager ein, nachdem von hier aus gegen eine illegale Siedlung vorgegangen worden war. 


\section{Wo steht der Widerstand heute?}

Für einige Monate haben die neuartigen kollektiven Aktionen den lange Zeit verschütteten Sinn für Solidarität wiederbelebt und die Zusammenarbeit von Gruppen der Gesellschaft ermöglicht, die sich traditionell als Gegner verstanden. Sie haben auch den Sinn für ein soziales Kollektiv befördert, das als Alternative $\mathrm{zu}$ atomisierten und konkurrierenden Individuen gesehen wurde. Dies stärkte gleichzeitig die Wahrnehmung der öffentlichen Sphäre als eines Raums, in dem es möglich schien, die sozialen Beziehungen neu zu gestalten, durch die Produktion neuer Diskurse eine andere Politik durchzusetzen und neue urbane Räume zu schaffen, in denen das Soziale auf andere Art in Erscheinung tritt.

Neue Vorstellungen zur Bedeutung von Reichtum wurden formuliert. An die Stelle der bisherigen Bewunderung des damit verbundenen glanzvollen Lebens trat die Verachtung und Beschuldigung derer, die sich ungerechtfertigt einen erheblichen Anteil des gemeinsamen Wohlstandes aneignen. Manche sprechen von einem Prozess der Ernüchterung in Bezug auf die endlose Suche nach mehr und besseren materiellen Gütern, was gleichzeitig mit einer kritischeren Sichtweise des politischen Apparates und der Beteiligung an der öffentlichen Sphäre verbunden war. Ging es ihnen zuvor stets darum, einen immer größeren Anteil am Konsum zu erjagen, so hatten sie nun ein gesteigertes Bewusstsein der Komplexität der Gesellschaft und entwickelten eine Art Klassenbewusstsein. Sie sind nun eher in der Lage, individualistischen Erklärungen für Erfolg und Scheitern mit Skepsis zu begegnen und nach institutionellen Begrenzungen und Fallstricken zu fragen.

Dennoch gibt es bisher keinerlei institutionelle Änderungen in Bezug auf die Themen, die vom Widerstand aufgeworfen wurden. Während sich sämtliche Parlamentsabgeordneten, ob Linke oder Rechte, auf den Diskurs der sozialen Gerechtigkeit beziehen, wurde praktisch nichts davon umgesetzt. Dazu kommt, dass der Widerstand einen Rückschlag erlitten hat. Einer der Gründe dafür ist die wirtschaftliche Rezession, die sich auf verschiedenen Ebenen manifestiert: in einem Rückgang des Konsums, einem Zurückfahren der Werbebudgets (die Unternehmen gehen davon aus, dass kostspielige Werbekampagnen ihnen auch keinen neuen Absatz bescheren werden) und der zunehmenden Gefahr von Entlassungen. Wie der Finanzminister es formuliert hat: Die europäische Wirtschaftskrise ist auf dem Weg nach Israel.

Ein anderer Grund für das Erlahmen des Widerstands ist - wie zu erwarten war - die wachsende Gegenbewegung von Seite des radikalen rechten Flügels, der unermüdlich dagegen ankämpft, wenn seine dominante Rolle und Ideologie in Frage gestellt wird. Seit den Protestaktionen wurden dem Parlament eine Reihe von rassistischen und anti-demokratischen Gesetzesvorhaben unterbreitet (und einige davon wurden sogar zustimmend diskutiert). Es ist demnach an der 
Zeit, dass der neue Diskurs und die kollektiven Aktionen in politische Macht umgesetzt werden, da sie sonst Gefahr laufen, unterzugehen.

Der Prozess, in dem neue Diskurse zu Gerechtigkeit und einem möglicherweise machtvolleren Kollektiv geführt wurden, hat die Palästinenser ausgegrenzt. Es steht daher immer noch an herauszufinden, wie ein sinnvoller Diskurs zu sozialer Gerechtigkeit und neuer Solidarität zwischen den Israelis damit verbunden werden kann, die langjährig und systematisch betriebenen Ungerechtigkeiten gegenüber den Palästinensern zu überwinden. Wird es möglich sein, sie in diesen Diskurs einzubinden und ihre Rechte anzuerkennen?

Übersetzung aus dem Englischen von Dorothea Schmidt

\section{Literatur}

Divided we stand: Why Inequality Keeps Rising (2011). OECD report from December 2011 (www. oecd.org/dataoecd/40/12/49170449.pdf, download 5.12.2011).

Hardt, Michael; Negri, Antonio (2004): Multitude: War and Democracy in the Age of Empire, New York.

Polanyi, Karl (2001): The Great Transformation: The Political and Economic Origins of Our Time, Boston.

Shalev, Michael; Grinberg, Lev Luis (1989): Histradut-government relations and the transition from Likud to a National Unity government: continuity and change in Israel's economic crises. Pinhas Spir Center for Development, Tel Aviv.

Yiftachel, Oren (2006): Ethnocracy: Land and Identity Politics in Israel/Palestine, Philadelphia. PEOPLE DEMAND ...

EIN JAHR

ARABISCHE REVOLUTIONEN FRAGEN AN DIE LINKE

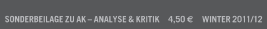

THE

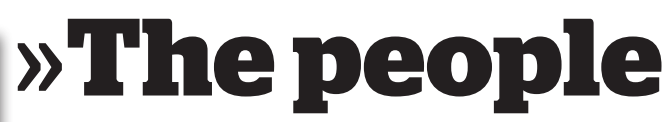
demand..." Ein Jahr arabische Revolutionen Fragen an die Linke. Ein als-Sonderheft.

Mit Beiträgen von Helmut Dietrich, Bernhard Schmid, Juliane Schumacher, Pedram Shahyar, Hannah Wettig u.a.

64 Seiten, $€ \mathbf{4 , 5 0}$ (+ Porto). Buchläden und WiederverkäuferInnen erhalten Rabatt. Bestellungen: vertrieb@akweb.de oder analyse \& kritik, Rombergstr. 10, 20255 Hamburg 